\title{
Epidemiology of violence in pediatric and adolescent nasal fracture compared with adult nasal fracture: An 8-year study
}

Hahyun $\mathrm{Yu}^{1}$,

Minseok Jeon ${ }^{1,2}$,

Youngjun Kim ${ }^{1}$,

Youngwoong Choi ${ }^{1}$

${ }^{1}$ Department of Plastic and

Reconstructive Surgery, Sanggye Paik

Hospital, Inje University College of

Medicine, Seoul; ${ }^{2}$ Anjung Public Health

Center, Pyeongtaek, Korea
Background: The epidemiology of nasal fractures varies according to factors such as the era and area of the study, as well as the age of the patient. We compared the characteristics and causes of pediatric nasal fractures.

Methods: A total of 2,321 patients with nasal fractures from 2010 to 2017 were examined. The patients were divided into age groups using the Korean school system of age classification. The causes of injury were divided into five groups: violence, fall or slip down, sports, road traffic accidents, and others. Fractures were classified using the Stranc and Robertson standard: vector of force and plane of fracture.

Results: Violence was the most common cause of nasal fracture in patients older than 12 years. Violence was a significantly less frequent cause among patients younger than 12 years old than among adolescent and adult patients. Nasal fractures due to violence were not observed in patients younger than 10 years. Plane 2 and lateral force fractures were the most common; however, in patients younger than 12 years, frontal force fractures were significantly more frequent than were lateral force fractures.

Conclusion: As children may simply be injured due to a fall or slip down, it is important for the parents and guardians to ensure their safety. As they become older, children should abstain from violence and be monitored. It is therefore very important to ensure that the environment is free of violence in order to prevent such injuries.

Keywords: Epidemiology / Fractures, bone / Nasal bone / Pediatrics

\section{INTRODUCTION}

Nasal bones can easily fracture from various injuries incurred during violent contact, sport activities, road traffic accidents, slips or falls, and other causes [1]. A relatively small amount of energy is sufficient to lead to a nasal fracture, as there is only a thin layer of skin with little fatty tissue above the nasal bones.

\section{Correspondence: Youngwoong Choi}

Department of Plastic and Reconstructive Surgery, Sanggye Paik Hospital, Inje

University College of Medicine, 1342 Dongil-ro, Nowon-gu, Seoul 01757, Korea

E-mail: pshero2@naver.com

Received July 17, 2019 / Revised July 29, 2019 / Accepted July 30, 2019
Even though nasal fractures are among the most common facial fractures in the pediatric and adult population [2], few studies have compared the characteristics of nasal fractures between children and adults. There are several anatomic differences between the pediatric and adult nasal bone; children's nasal bones have a far less pronounced projection and many more cartilage components. These anatomical differences result in different nasal fracture characteristics among the various age groups.

There are many epidemiological articles on the natural incidence of nasal fractures. As a small amount of energy can cause 
a nasal fracture, its causes and frequency patterns vary according to the era and area of study. In addition, the age of the patient is also an important component of such epidemiological studies. Here, we compared the characteristics and causes of pediatric nasal fractures.

\section{METHODS}

We reviewed the medical records of patients with nasal fractures from January 2010 to December 2017 in a single institute in Seoul, Korea. All patients, even those with previous history of facial trauma or cosmetic surgery, were included. Patients with nasal fractures alongside other facial bone fractures were also included. Based on these criteria, a total of 2,321 patients were included in this study. Data obtained from medical records and computed tomography included patient's age, gender, cause of trauma, and the classification of nasal fracture.

Causes of injury were divided into five groups: violence, fall or slip down, sports, road traffic accidents, and others. Patients were divided into five groups according to their age using the Korean school system of age classification. Group 1 consisted of preschool children 0-5 years old; group 2, of elementary school-age children 6-11 years old; group 3, of middle-school age children 12-14 years old; group 4, of high-school age children 15-17 years old; and group 5 consisted of adults older than 18 years of age.

Fractures were classified using the standard by the Stranc and Robertson [3]. Following this standard, nasal fractures were classified by the vector of force (frontal impact and lateral force) and the plane of the fracture (planes 1,2, and 3). Computed tomography images were used for the classification, and several views including the axial, coronal, and sagittal, as well as 3-dimensional images, were used.

The ratio of each factor of each group was compared with the adult group (group 5). The independent $t$-test and Pearson chisquare test were used. All statistical analyses were performed using the SPSS software version 18.0 (SPSS Inc., Chicago, IL, USA). For all analyses, significance was set at $p<0.05$.

\section{RESULTS}

From January 2010 to December 2017, a total of 2,321 patients received surgical intervention for a nasal fracture. Patients were classified by age according to the Korean school system of age classification (see methods). Groups 1 to 4 included 44, 125, 180 , and 322 patients, respectively, whereas group 5 included 1,650 adult patients older than 18 years. Of the 2,321 patients, 1,805 were male and 516 were female. The oldest patient was an 89-year-old male, and the youngest was a 9-month-old female.

The most common cause of injury in the total population was violence, affecting 715 patients (31\%), followed by a fall or slip down (647 patients, $28 \%$ ), sports (354 patients, $15 \%$ ), road traffic accidents (323 patients, 14\%), and other factors (282 patients, 12\%). In groups 1 and 2, fall or slip down was the most common cause of injury, while in groups 3,4 , and 5 , violence was the most common cause of injury (Table 1).

We further investigated the proportion of nasal fractures resulting from violence by age. Nasal fracture due to violence was first observed from the age of 10 years, with the rate being highest at 13 years old (41.38\%) (Fig. 1). The ratio of violence in adults was 531 of 1,650 (32.18\%). In group 1, none of the $44 \mathrm{pa}-$ tients were injured by violence; in group 2 , nine of 125 patients (7.20\%) had nasal bone injuries due to violence. In group 3, 69 of $180(38.33 \%)$ reported cases were caused by violence, and this was the highest among the five reported groups. In group 5, the proportion of violence as a contributing factor was 531 of

Table 2. Number and proportion of violence by group according to the Korean school system classification of age

\begin{tabular}{lcrcr}
\hline Group & Age (yr) & Total & Violence & $p$-value ${ }^{\text {a) }}$ \\
\hline 1. Preschool & $0-5$ & 44 & 0 & $<0.001$ \\
2. Elementary school & $6-11$ & 125 & $9(7.2)$ & $<0.001$ \\
3. Middle school & $12-14$ & 180 & $69(38.3)$ & 0.112 \\
4. High school & $15-17$ & 322 & $106(32.9)$ & 0.841 \\
5. Adult & $\geq 18$ & 1,650 & $531(32.2)$ & \\
\hline
\end{tabular}

Values are presented as number (\%).

${ }^{\text {a) }}$ Chi-square test. The statistical result was calculated by comparison with the adult group.

Table 1. Causes of injury in a nasal fracture

\begin{tabular}{lcccccc}
\hline Group & Age $(\mathrm{yr})$ & Violence & Fall or slip & Sports & \multicolumn{2}{c}{ Road TA } \\
\hline 1. Preschool & $0-5$ & 0 & $29(65.91)$ & $1(2.27)$ & $6(13.64)$ & Others \\
2. Elementary school & $6-11$ & $9(7.20)$ & $70(56.00)$ & $27(21.60)$ & $6(4.80)$ & $13(10.40)$ \\
3. Middle school & $12-14$ & $69(38.33)$ & $23(12.78)$ & $52(28.89)$ & $11(6.11)$ & $25(13.89)$ \\
4. High school & $15-17$ & $106(32.92)$ & $38(11.80)$ & $110(34.16)$ & $38(11.80)$ & $30(9.32)$ \\
5. Adult & $\geq 18$ & $531(32.18)$ & $487(29.52)$ & $164(9.94)$ & $262(15.88)$ & $206(12.48)$ \\
\hline
\end{tabular}

Values are presented as number (\%).

TA, traffic accident. 


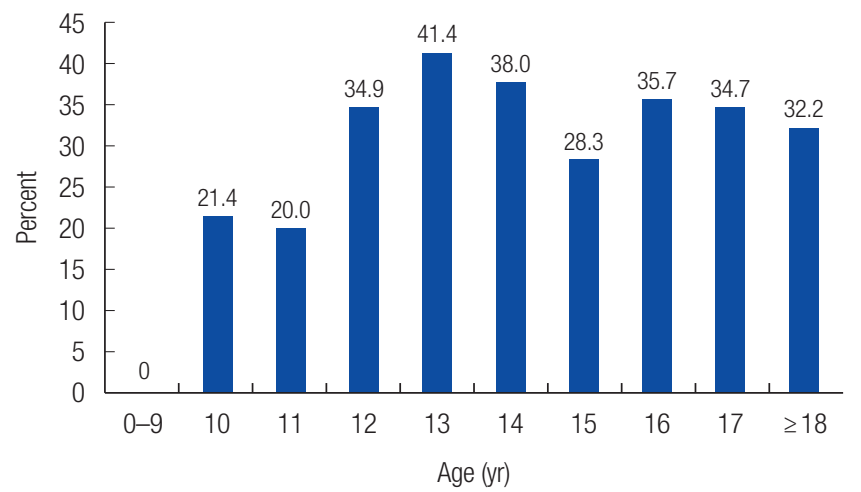

Fig. 1. Proportion of violence in 1-year intervals of age.

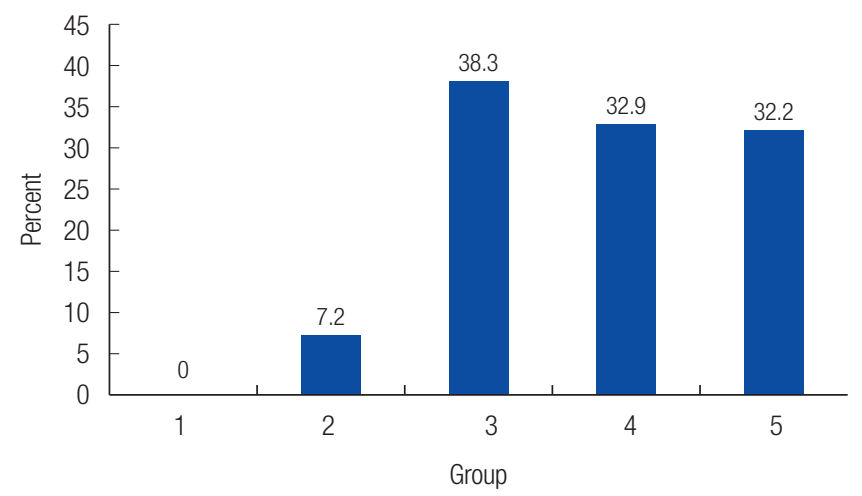

Fig. 2. Proportion of violence by age group according to the Korean school system classification of age. Group 1, preschool; group 2, elementary school; group 3, middle school; group 4, high school; group 5, adult.

Table 3. Classification by vector of force (frontal impact and lateral force) and plane of fracture (planes 1, 2, and 3) in nasal fractures

\begin{tabular}{|c|c|c|c|c|c|c|c|c|}
\hline \multirow{2}{*}{ Group } & \multirow{2}{*}{ Age (yr) } & \multicolumn{3}{|c|}{ Frontal impact } & \multicolumn{3}{|c|}{ Lateral force } & \multirow{2}{*}{$p$-value $e^{a}$} \\
\hline & & Plane 1 & Plane 2 & Plane 3 & Plane 1 & Plane 2 & Plane 3 & \\
\hline 1. Preschool & $0-5$ & $5(11.36)$ & $27(61.36)$ & 0 & $1(2.27)$ & $10(22.73)$ & $1(2.27)$ & 0.001 \\
\hline 2. Elementary school & $6-11$ & $19(15.20)$ & $52(41.60)$ & 0 & $6(4.80)$ & $47(37.60)$ & $1(0.8)$ & 0.001 \\
\hline 3. Middle school & $12-14$ & $14(7.78)$ & $42(23.33)$ & $6(3.33)$ & $6(3.33)$ & $107(59.44)$ & $5(2.78)$ & 0.825 \\
\hline 4. High school & $15-17$ & $37(11.49)$ & $66(20.50)$ & $5(1.55)$ & $10(3.11)$ & 191 (59.32) & $13(4.04)$ & 0.551 \\
\hline 5. Adult & $\geq 18$ & $156(9.45)$ & 390 (23.64) & $36(2.18)$ & $47(2.85)$ & 909 (55.09) & $112(6.79)$ & \\
\hline
\end{tabular}

Values are presented as number (\%).

${ }^{a}$ Chi-square test. The statistical result was calculated by comparison with the frontal impact and the lateral force.

1,650 (32.18\%). A similar proportion was observed in group 4, while group 3 had a slightly lower proportion (Table 2, Fig. 2). In groups 1 and 2 , the violence rate was significantly lower than was in the adult group $(p=0.000)$. In group 3 , the proportion of violence was much higher than it was in group 5; however, the difference was not significant $(38.3 \%$ vs. $32.2 \%, p=0.112)$. No differences were observed between groups 4 and 5 (32.9\% vs. $32.2 \%, p=0.845$ ).

Nasal fractures were classified using the Stranc-Roberson standard (Table 3). The most common type observed was plane 2 with lateral force, affecting 1,264 of the patients (54.46\%), followed by plane 2 with frontal impact and plane 1 with frontal impact. Lateral force (1,466 patients, $63.16 \%)$ was more common than was frontal impact (855 patients, 36.84\%). In contrast, frontal impact was significantly higher than was lateral force in groups 1 and 2 ( $p=0.001$ and $p=0.001$, respectively). Of all, plane 3 had the lowest incidence rate, including both frontal impact and lateral force. There was no significant connection between the forces and causes of the nasal fractures.

\section{DISCUSSION}

The nose is one of the most frequently damaged structures of the body, as it is the most exposed feature of the face. Fracture of the nose is the most common type of facial fracture and the third most common fracture of the human skeleton [4]. In the pediatric population, a nasal fracture is also the most common facial bone fracture [2]. The nasal bone is located at the center of the mid-facial area and has a plate-like structure protruding forward [1]. The bones and the skin are in close proximity, and the fat layer, that usually functions as a buffer, is relatively thin. As a result, a nasal fracture can occur with a relatively weak impact.

Nasal fractures are less frequent at a younger age $[5,6]$. Hwang et al. [1] from Korea reported that only $3.9 \%$ of all nasal fractures occurred under the age of 10 years. In our study, the incidence rates of nasal fractures in groups 1 and 2, i.e., those including patients under the age of 12 years, were $44(1.9 \%)$ and 125 (9.5\%), respectively. As the age increased, the number of patients with nasal fracture increased, with its highest peak at 16 years of age (115 patients, 4.95\%). Kim et al. [7] from Korea also reported that facial fractures occurred most frequently in individuals aged 13 to 15 years.

There have been numerous epidemiology and etiology studies on nasal fractures due to its high occurrence rate due to various reasons including violence, sport, fall, or slip injuries; and traffic 
accidents. Hwang et al. [1] reported that the most common cause of nasal injury in children and adults is fighting, whereas Basheeth et al. [8] reported that accident-dominant injury was the most common cause of nasal fracture (40.8\%). Several studies have also examined the variation of nasal fractures by race and period $[9,10]$. However, there are no absolute standards for the etiology and epidemiology of nasal fractures, as results vary depending on the location and timing of the study. Moran [11] suggested that infants frequently bump into objects, pull objects onto their face, and sustain numerous falls. These childhood factors lead to a difference in the epidemiology of nasal fractures between adults and children [11]. Due to the different mechanisms of injuries, the radiologic features of nasal fracture also differ between the pediatric and adult populations [12]. Gender also has a role in this difference [9].

Previous studies have reported that aggression (violence) is the most common cause of nasal fracture in the general population [13]. In groups 1 and 2, fall or slip down was the most common cause of injury and had a higher incidence rate than it did in groups 3, 4, and 5, although the difference was not significant. However, violence had a significantly lower incidence rate in groups 1 and 2 compared to the adult group (group 5). There were no reports of nasal fracture by violence under the age of 10 years in our study. With increasing age, sport-related injuries and violence were more common, while the incidence rate of accidental falls decreased. Kim et al. [7] also reported that the mean age of the patients involved in violence was 14.4 years. In our study, violence was the most common cause of nasal fracture in the middle-school aged group (group 3). The percentage of nasal fractures caused by violence in group 3 was much higher than that in the adult group, but the difference was not significant. The incidence of nasal fractures caused by violence in groups 1 and 2 was significantly lower than that in the adult (group 5) and adolescent (group 3) groups.

Exposure to violence has a negative impact on children's physical, emotional, and cognitive well-being [14]. Recently, violence in the pediatric population has become a serious social issue in Korea [15]. Not only violence but complications after trauma can have a negative impact on childhood. This study provides an overview of nasal fractures in the pediatric and adolescent age groups, which helps illustrate the trends and characteristics of these fractures.

To investigate the factors affecting childhood nasal fractures, we found a method that allows comparison with adult nasal fractures; the classification created by Stranc and Robertson [3]. In this classification, nasal fractures are divided by the vector of the force: frontal impact and lateral force. Lateral force fracture is the most common and has a good prognosis for both appear- ance and nasal airway function. Furthermore, lateral force fractures rarely cause disruption of the osseous and cartilaginous components of the nose. In our study, lateral force fractures were the most, especially of plane type 2 , but not in groups 1 and 2.

In our study, the incidence rate of frontal impact fractures was significantly higher than that of lateral force fractures in groups 1 and 2 compared to the adult group. After the age of 12 years, the incidence of lateral force fractures was significantly higher than that of frontal impact fractures. This pattern can be explained by the anatomical structure of the pediatric nasal bone. Children's noses are structurally different from those of adults, in the latter's case, consisting of bones and cartilage supporting the skeletal structure. The pediatric nose skeleton is composed of cartilage, rather than of bone, to a greater extent with reduced frontal projection. The lesser projection can explain why it is less likely for the pediatric nose to be damaged by lateral forces. The lateral nasal bone may also bend when lateral force is applied because of the nasal cavity. However, when frontal impact is applied, it is not easy to bend due to the nasal septum. In younger children, the nasal bones are also separated in the midline by an open suture line, and the nasal bones laterally overlap with the frontal processes of the maxilla [16]. Frontal nose's soft tissue and soft cartilage projection bend easily during facial trauma. However, when classified by plane, adults were more likely to have a higher incidence rate of nasal fracture compared to the pediatric groups, i.e., significantly higher plane 2 fractures of both frontal impact and lateral force. This is because nasal fracture itself has a lower incidence rate in the pediatric population.

The nasal septum has two growth centers, which influence midface growth and development. The sphenodorsal zone, which extends from the sphenoid to the nasal dorsum, is believed to be responsible for the growth of the length and height of the nasal dorsum. Conversely, the sphenospinal zone, which extends from the sphenoid to the anterior nasal spine, appears to control the outgrowth of the maxilla [17]. Septal injury may cause permanent facial deformity by disrupting the growth of the center of the face [18]. In groups 1 and 2, frontal impact was more dominant than was lateral force. Children are more susceptible to frontal impact, which directly affects the nasal tip cartilage as well as the septal cartilage [3]. Kim et al. [19] reported that $46.9 \%$ of nasal fractures occur in combination with septal fractures. It is important to distinguish among nasal fractures because injury to the cartilaginous framework frequently requires a secondary procedure [3].

This study only accounted for patients who underwent surgery for a nasal fracture, and their long-term follow-up data 
were not available. Patients with nasal fracture who did not undergo operation were not included. Further studies are required including non-operated patients. Long-term follow-up data will also provide additional insights.

Nasal fractures in the pediatric population present unique challenges. Pediatric nasal fractures can result in underlying injury on the cartilage framework, which frequently requires a secondary procedure. Pediatric nasal fracture also has a much shorter interval available between the injury and the operation compared to adult nasal fracture. Therefore, it is important to rapidly diagnose a nasal fracture. As children may simply be injured due to a fall or slip, it is important for parents and guardians to ensure their safety. As they become older, children should abstain from violence and be monitored. The epidemiology and characteristics of nasal fracture vary depending on the social environment.

\section{NOTES}

\section{Conflict of interest}

No potential conflict of interest relevant to this article was reported.

\section{Ethical approval}

The study was performed in accordance with the principles of the Declaration of Helsinki. Written informed consents were obtained.

\section{ORCID}

Hahyun Yu https://orcid.org/0000-0002-0454-5070

Minseok Jeon https://orcid.org/0000-0002-6700-6971

Youngjun Kim https://orcid.org/0000-0001-7797-248X

Youngwoong Choi https://orcid.org/0000-0002-2211-2981

\section{REFERENCES}

1. Hwang K, You SH, Kim SG, Lee SI. Analysis of nasal bone fractures; a six-year study of 503 patients. J Craniofac Surg 2006;17: 261-4.

2. Lee WT, Koltai PJ. Nasal deformity in neonates and young children. Pediatr Clin North Am 2003;50:459-67.

3. Stranc MF, Robertson GA. A classification of injuries of the nasal skeleton. Ann Plast Surg 1979;2:468-74.

4. Murray JA, Maran AG, Mackenzie IJ, Raab G. Open v closed reduction of the fractured nose. Arch Otolaryngol 1984;110:
797-802.

5. Ul Haq ME, Khan AS. A retrospective study of causes, management, and complications of pediatric facial fractures. Eur J Dent 2018;12:247-52.

6. Zimmermann CE, Troulis MJ, Kaban LB. Pediatric facial fractures: recent advances in prevention, diagnosis and management. Int J Oral Maxillofac Surg 2005;34:823-33.

7. Kim SH, Lee SH, Cho PD. Analysis of 809 facial bone fractures in a pediatric and adolescent population. Arch Plast Surg 2012; 39:606-11.

8. Basheeth N, Donnelly M, David S, Munish S. Acute nasal fracture management: a prospective study and literature review. Laryngoscope 2015;125:2677-84.

9. Hwang K, Ki SJ, Ko SH. Etiology of nasal bone fractures. J Craniofac Surg 2017;28:785-8.

10. Liu C, Legocki AT, Mader NS, Scott AR. Nasal fractures in children and adolescents: mechanisms of injury and efficacy of closed reduction. Int J Pediatr Otorhinolaryngol 2015;79:223842.

11. Moran W. Nasal trauma in children. Otolaryngol Clin North Am 1977;10:95-101.

12. Yabe T, Tsuda T, Hirose S, Ozawa T. Comparison of pediatric and adult nasal fractures. J Craniofac Surg 2012;23:1364-6.

13. Fornazieri MA, Yamaguti HY, Moreira JH, Navarro PL, Heshiki RE, Takemoto LE. Fracture of nasal bones: an epidemiologic analysis. Int Arch Otorhinolaryngol 2008;12:498-501.

14. Kashani JH, Daniel AE, Dandoy AC, Holcomb WR. Family violence: impact on children. J Am Acad Child Adolesc Psychiatry 1992;31:181-9.

15. Kim DH, Kim KI, Park YC, Zhang LD, Lu MK, Li D. Children's experience of violence in China and Korea: a transcultural study. Child Abuse Negl 2000;24:1163-73.

16. Alcaraz N, Lawson W. Trauma of the nose and nasoethmoid complex in children and adolescents. Facial Plast Surg Clin North Am 1999;7:175-84.

17. Verwoerd CD, Verwoerd-Verhoef HL. Rhinosurgery in children: developmental and surgical aspects of the growing nose. Laryngorhinootologie 2010;89(Suppl 1):S46-71.

18. Bhuskute A, Sumiyoshi M, Senders C. Septorhinoplasty in the pediatric patient. Facial Plast Surg Clin North Am 2016;24:24553.

19. Kim JE, Park HS, Yoon CH, Kim HJ. Analysis of nasal septal fracture combined in nasal bone fracture using computerized tomography. J Korean Soc Plast Reconstr Surg 1998;25:852-9. 